\title{
High-Temperature Heat Capacity \\ and Thermodynamic Properties \\ of Oxide Compounds of $\mathrm{Bi}_{2} \mathrm{O}_{3}-\mathrm{P}_{2} \mathrm{O}_{5}$
}

\author{
Liubov T. Denisova*, \\ Liubov G. Chumilina, Nikolai A. Babitskiy, \\ Vladimir P. Zhereb and Viktor M. Denisov \\ Siberian Federal University \\ 79 Svobodny, Krasnoyarsk, 660041 Russia
}

Received 09.01.2015, received in revised form 11.02.2015, accepted 28.02.2015

The high temperature heat capacity of $\mathrm{Bi}_{24} \mathrm{P}_{2} \mathrm{O}_{41}$ (336-1073K) and $\mathrm{Bi}_{14} \mathrm{P}_{4} \mathrm{O}_{31}$ (394-1121 K) has been investigated using differential scanning calorimetry. The experimental heat capacities were used to calculate the thermodynamic functions of this compounds (enthalpy change and entropy change).

Keywords: bismuth oxide, phosphorus oxide, heat capacity, thermodynamic properties.

\section{Теплоемкость и термодинамические свойства оксидных соединений системы $\mathrm{Bi}_{2} \mathrm{O}_{3}-\mathrm{P}_{2} \mathrm{O}_{5}$}

Л.Т. Денисова, Л.Г. Чумилина, Н.А. Бабицкий, В.П. Жереб, В.М. Денисов

Сибирский федеральный университет Россия, 660041, Красноярск, пр. Свободный,79

Методом дифференциальной сканирующей калориметрии измерена молярная теплоемкость $\mathrm{Bi}_{24} \mathrm{P}_{2} \mathrm{O}_{41}(336-1073 \mathrm{~K})$ и $\mathrm{Bi}_{14} \mathrm{P}_{4} \mathrm{O}_{31}(394-1121 \mathrm{~K})$. Величины термодинамических функций (изменения энтальпии и энтропии) рассчитаны по сглаженным значениям теплоемкости.

Ключевые слова: оксид висмута, оксид фосфора, теплоемкость, термодинамические свойства.

(C) Siberian Federal University. All rights reserved

* Corresponding author E-mail address: antluba@mail.ru 


\section{Введение}

Поиск и создание новых функциональных материалов, обладающих ценными для практических приложений свойствами, - одна из актуальных задач современного материаловедения. Особое место среди таких материалов занимают кристаллы и стекла на основе $\mathrm{Bi}_{2} \mathrm{O}_{3}$ [1-3]. Несмотря на такое внимание к подобным соединениям, некоторые из них еще недостаточно исследованы. К ним относятся оксидные соединения системы $\mathrm{Bi}_{2} \mathrm{O}_{3}-\mathrm{P}_{2} \mathrm{O}_{5}$. О сложности их получения могут свидетельствовать разные варианты фазовых отношений в системе $\mathrm{Bi}_{2} \mathrm{O}_{3}-\mathrm{P}_{2} \mathrm{O}_{5}$, которые различаются как по составу образующихся соединений, так и по их количеству $[1$, 4-8]. В то же время для оптимизации условий получения соединений необходимо термодинамическое изучение фаз, которое возможно лишь при наличии сведений об их термодинамических свойствах. Такие данные для системы $\mathrm{Bi}_{2} \mathrm{O}_{3}-\mathrm{P}_{2} \mathrm{O}_{5}$ отсутствуют.

Цель настоящей работы - исследование высокотемпературной теплоемкости $\mathrm{Bi}_{24} \mathrm{P}_{2} \mathrm{O}_{41}$ и $\mathrm{Bi}_{14} \mathrm{P}_{4} \mathrm{O}_{31}$ и определение по этим данным их термодинамических свойств.

\section{Экспериментальная часть}

Сложность получения оксидных соединений $\mathrm{Bi}_{2} \mathrm{O}_{3}-\mathrm{P}_{2} \mathrm{O}_{5}$ связана с тем, что многие из них плавятся инконгруэнтно и могут быть получены либо твердофазным синтезом, либо из раствора-расплава. Соединение $\mathrm{Bi}_{24} \mathrm{P}_{2} \mathrm{O}_{41}$ получено из $\mathrm{Bi}_{2} \mathrm{O}_{3}$ (ос.ч) и $\mathrm{BiPO}_{4}$ (хч) методом твердофазного синтеза подобно [4] (температура $1020 \mathrm{~K}$, время отжига - 320 ч, два промежуточных перетирания). Контроль полученных образцов проводили с использованием рентгенофазового анализа (X'Pert Pro фирмы «PANalytical», Нидерланды; излучение $\mathrm{CuK}_{\alpha}$ ). Отметим, что на дифрактограммах присутствовали только рефлексы, отвечающие соединению $\mathrm{Bi}_{24} \mathrm{P}_{2} \mathrm{O}_{41}$ (рис. 1). Параметр решетки $a=10.15117(6) \AA$, что близко к данным [1], где $a=10.1691 \AA$.

Монокристаллы $\mathrm{Bi}_{14} \mathrm{P}_{4} \mathrm{O}_{31}$ выращены из раствора-расплава $\mathrm{Bi}_{2} \mathrm{O}_{3}-\mathrm{BiPO}_{4}-\mathrm{BPO}_{4}-\mathrm{B}_{2} \mathrm{O}_{3}$ намораживанием на платиновую проволочку с последующим выделением в процессе роста монокристаллического образца. Параметры структуры выращенного монокристалла хорошо совпали с результатами, приведенными в работе [9].

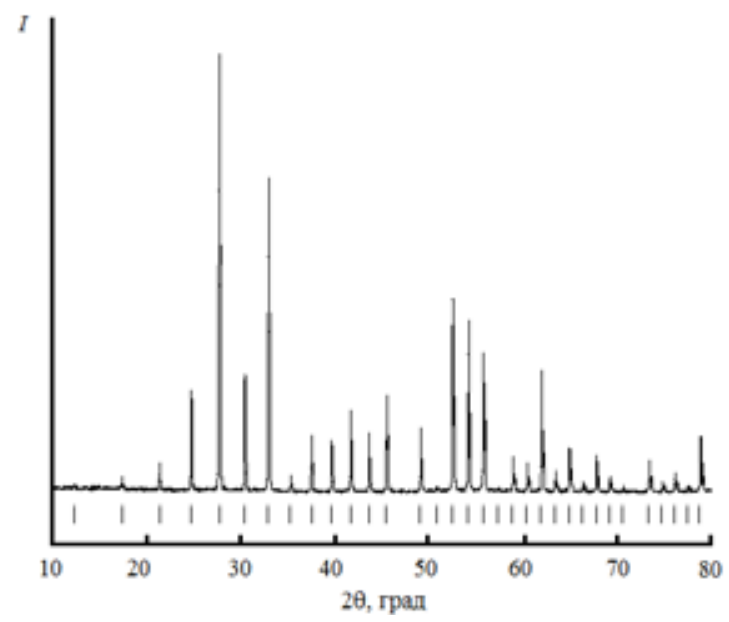

Рис. 1. Дифрактограмма $\mathrm{Bi}_{24} \mathrm{P}_{2} \mathrm{O}_{41}$ при комнатной температуре 
Измерение молярной теплоемкости поликристаллического $\mathrm{Bi}_{24} \mathrm{P}_{2} \mathrm{O}_{41}$ и монокристалла $\mathrm{Bi}_{14} \mathrm{P}_{4} \mathrm{O}_{31}$ проводили методом дифференциальной сканирующей калориметрии на приборе STA 449 C Jupiter (NETZSCH) с использованием специальных держателей для измерения теплоемкости. Методика измерений $C_{p}$ подобна описанной ранее [10,11]. Исследованные интервалы температур выбраны экспериментально на основе дифференциального термического анализа (STA 449 C). Экспериментальные результаты обрабатывались с применением пакета анализа NETZSCH Proteus Thermal Analysis и лицензионного программного инструмента Systat Sigma Plot 12.

Получение соединений $\mathrm{Bi}_{24} \mathrm{P}_{2} \mathrm{O}_{41}$ и $\mathrm{Bi}_{14} \mathrm{P}_{4} \mathrm{O}_{31}$ и измерение их высокотемпературной теплоемкости проводили в платиновых тиглях.

\section{Обсуждение результатов}

На рис. 2 (кривая 1) приведены данные по влиянию температуры на молярную теплоемкость $\mathrm{Bi}_{24} \mathrm{P}_{2} \mathrm{O}_{41}$.

Видно, что при росте температуры от 336 до $1073 \mathrm{~K}$ значения $C_{p}$ закономерно увеличиваются, а на зависимости $C_{p}=f(T)$ нет различного рода экстремумов. Полученные значения $C_{p}$ в исследованном интервале температур могут быть описаны уравнением Майера - Келли [12]

$$
C_{p}=a+b T+c T^{-2}=1562.2+173.0 \cdot 10^{-3} T-209.0 \cdot 10^{5} T^{-2} .
$$

Коэффициент корреляции для уравнения (1) равен 0.9988.

$\mathrm{C}$ использованием соотношения (1) по известным термодинамическим уравнениям рассчитаны термодинамические функции (изменение энтальпии $H^{0}(T)-H^{0}(336 \mathrm{~K})$ и энтропии $\left.S^{0}(T)-S^{0}(336 \mathrm{~K})\right)$. Полученные данные приведены в табл. 1.

Из этой таблицы следует, что при температурах выше $800 \mathrm{~K}$ теплоемкость превышает классический предел Дюлонга - Пти $3 R s$, где $R$ - универсальная газовая постоянная, $s$ - число атомов в формульной единице $\mathrm{Bi}_{24} \mathrm{P}_{2} \mathrm{O}_{41}(s=67)$.

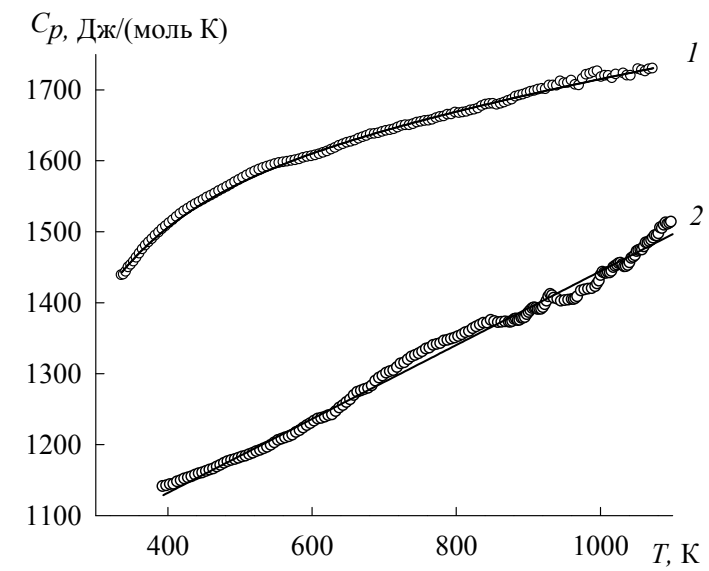

Рис. 2. Зависимость теплоемкости $\mathrm{Bi}_{24} \mathrm{P}_{2} \mathrm{O}_{41}(1)$ и $\mathrm{Bi}_{14} \mathrm{P}_{4} \mathrm{O}_{31}$ (2) от температуры: точки - экспериментальные данные; линия - аппроксимирующая кривая

$$
-139-
$$


Таблица 1. Сглаженные значения теплоемкости и рассчитанные по ним термодинамические функции $\mathrm{Bi}_{24} \mathrm{P}_{2} \mathrm{O}_{41}$

\begin{tabular}{|c|c|c|c|}
\hline$T, \mathrm{~K}$ & $\begin{array}{c}C_{p}, \\
\text { Дж/(моль К })\end{array}$ & $\begin{array}{c}H^{0}(T)-H^{0}(336 \mathrm{~K}), \\
\text { кДж/моль }\end{array}$ & $\begin{array}{c}S^{0}(T)-S^{0}(336 \mathrm{~K}), \\
\text { Дж/(моль К })\end{array}$ \\
\hline 336 & 1442 & - & - \\
350 & 1459 & 20.31 & 59.23 \\
400 & 1506 & 94.50 & 257.3 \\
450 & 1541 & 170.7 & 436.8 \\
500 & 1568 & 248.5 & 600.6 \\
550 & 1591 & 327.5 & 751.2 \\
600 & 1610 & 407.5 & 890.4 \\
650 & 1627 & 488.4 & 1020 \\
700 & 1642 & 570.2 & 1141 \\
750 & 1656 & 652.7 & 1255 \\
800 & 1669 & 735.8 & 1362 \\
850 & 1681 & 819.6 & 1464 \\
900 & 1693 & 903.9 & 1560 \\
950 & 1704 & 988.9 & 1652 \\
1000 & 1715 & 1074 & 1740 \\
1050 & 1726 & 1160 & 1824 \\
\hline
\end{tabular}

Считая в первом приближении, что $C_{p}$ и $C_{v}$ для $\mathrm{Bi}_{24} \mathrm{P}_{2} \mathrm{O}_{41}$ близки, а также используя найденное нами значение характеристической температуры Дебая $\Theta_{D}=585 \mathrm{~K}$ (определено по экспериментальным значениям теплоемкости для минимальных температур эксперимента) и таблицы функций Дебая $\left(\Theta_{D} / T\right)$ [13], мы рассчитали $C_{p}$. Установлено, что после $T=600 \mathrm{~K}$ появляется разница между экспериментальными и рассчитанными значениями $C_{p}$. Причем с ростом температуры это различие увеличивается и при $T=1000 \mathrm{~K}$ составляет $4,2 \%$. Это можно связать с тем, что значения $\Theta_{D}$, найденные для одного и того же вещества из экспериментальных данных при разных температурах, часто оказываются различными, то есть $\Theta_{D}$ является функцией температуры [13].

Влияние температуры на теплоемкость $\mathrm{Bi}_{14} \mathrm{P}_{4} \mathrm{O}_{31}$ в интервале температур 394-1121 K (рис. 2, кривая 2) хорошо описывается уравнением

$$
C_{p}=923.76+52.07 \cdot 10^{-2} T
$$

которое лучше, чем уравнение Майера-Келли, передает зависимость $C_{p}=f(T)$. Коэффициент корреляции для уравнения (2) равен $r=0.9958$.

С использованием уравнения (2) рассчитаны $H^{0}(T)-H^{0}(394 \mathrm{~K})$ и $S^{0}(\mathrm{~T})-S^{0}(394 \mathrm{~K})$. Полученные данные представлены в табл. 2. Можно отметить, что и для соединения $\mathrm{Bi}_{14} \mathrm{P}_{4} \mathrm{O}_{31}$ теория Дебая не описывает экспериментальные значения теплоемкости в зависимости от температуры. Это можно связать с тем, что теория Дебая плохо применима для многоатомных соединений [13].

\section{Выводы}

Исследовано влияние температуры на молярную теплоемкость $\mathrm{Bi}_{24} \mathrm{P}_{2} \mathrm{O}_{41}$ (336-1073 K) и $\mathrm{Bi}_{14} \mathrm{P}_{4} \mathrm{O}_{31}$ (394-1121 K). Установлено, что теория Дебая не описывает экспериментальные значе- 
Таблица 2. Сглаженные значения теплоемкости и рассчитанные по ним термодинамические функции $\mathrm{Bi}_{14} \mathrm{P}_{4} \mathrm{O}_{31}$

\begin{tabular}{|c|c|c|c|}
\hline$T, \mathrm{~K}$ & $\begin{array}{c}C_{p}, \\
\text { Дж/(моль К })\end{array}$ & $\begin{array}{c}H^{0}(T)-H^{0}(394 \mathrm{~K}), \\
\text { кДж/моль }\end{array}$ & $\begin{array}{c}S^{0}(T)-S^{0}(394 \mathrm{~K}), \\
\text { Дж/(моль К })\end{array}$ \\
\hline 394 & 1129 & - & - \\
400 & 1132 & 6.78 & 17.09 \\
450 & 1158 & 64.04 & 151.9 \\
500 & 1184 & 122.6 & 275.3 \\
550 & 1210 & 182.4 & 389.4 \\
600 & 1236 & 243.6 & 495.8 \\
650 & 1262 & 306.1 & 595.8 \\
700 & 1288 & 369.8 & 690.2 \\
750 & 1314 & 434.9 & 780.0 \\
800 & 1340 & 501.3 & 865.7 \\
850 & 1366 & 568.9 & 947.7 \\
900 & 1392 & 637.9 & 1026 \\
1000 & 1418 & 708.2 & 1103 \\
1050 & 1444 & 779.7 & 1176 \\
1100 & 1470 & 852.6 & 1247 \\
\hline
\end{tabular}

ния теплоемкости во всем исследованном интервале температур. Рассчитаны термодинамические свойства оксидных соединений.

Работа выполнена в рамках государственного задания Минобрнауки России Сибирскому федеральному университету.

\section{Список литературы}

1. Ю.Ф. Каргин, В.И. Бурлаков, А.А. Марьин и др. Кристаллы $\mathrm{Bi}_{12} \mathrm{M}_{\mathrm{x}} \mathrm{O}_{20 \pm \delta}$ со структурой силленита. Синтез, строение, свойства. М.: ИОНХ, 2004. 316 с. [Kargin Y.F., Burkov V.I., Maryin A.A. et al. Crystals $\mathrm{Bi}_{12} \mathrm{M}_{\mathrm{x}} \mathrm{O}_{20 \pm \delta}$ with a structure of sillenite. Synthesis, structure, properties. Moscow, IONKh. 2004. 316 p. (In Russ.)]

2. Денисов В.М., Белоусова Н.В., Моисеев Г.К. и др. Висмутсодержащие материалы: строение и физико-химические свойства. Екатеринбург: УрО РАН, 2000. 526 c. [Denisov V.M., Belousova N.V., Moiseev G.K. et al. Materials bismuth-containing: structure and physicochemical properties. Ekaterinburg. UrO RAN. 2006. 526 p. (In Russ.)]

3. Юхин Ю.М., Михайлов Ю.И. Химия висмутовых соединений и материалов. Новосибирск: СО РАН, 2001. 360 с. [Yuhin Yu.M., Mihailov Yu.I. Chemistry of bismuth-containing compounds and materials. Novosibirsk: SO RAN, 2001. 360 p. (In Russ.)].

4. Волков В.В., Жереб Л.А., Каргин Ю.Ф. и др. Система $\mathrm{Bi}_{2} \mathrm{O}_{3}-\mathrm{P}_{2} \mathrm{O}_{5} / /$ ЖНХ. 1983. Т. 28. № 4. C. 1002 - 1005. [Volkov V.V., Zhereb V.P, Kargin Yu.F. et. al. System of $\mathrm{Bi}_{2} \mathrm{O}_{3}-\mathrm{P}_{2} \mathrm{O}_{5} / /$ Zhurnal neorganicheskoi khimii. 1983. V. 28. № 4. C. 1002 - 1005. (In Russ.)]

5. Воеводский В.Ю., Каргин Ю.Ф., Скориков В.М. Система $\mathrm{Bi}_{2} \mathrm{O}_{3}-\mathrm{P}_{2} \mathrm{O}_{5}$ в области концентраций 50 - 85 мол. \% $\mathrm{P}_{2} \mathrm{O}_{5} / /$ ЖНХ. 1997. Т. 42. № 5. С. 800 - 802 [Voevodskii V.Yu., Kargin 
Yu.F., Skorikov V.M. System $\mathrm{Bi}_{2} \mathrm{O}_{3}-\mathrm{P}_{2} \mathrm{O}_{5}$ in composition range 50-85 mol \% $\mathrm{P}_{2} \mathrm{O}_{5} / /$ Russian Journal of Inorganic Chemistry. 1997. V. 42. № 5. Р. 715-717].

6. Жереб В.П., Каргин В.П., Жереб Л.А. и др. Стабильные и метастабильные равновесия в системе $\mathrm{Bi}_{2} \mathrm{O}_{3}-\mathrm{BiPO}_{4} / /$ Неорган. материалы. 2003. T. 39. № 8. С. 999 - 1002 [Zhereb V.P., Kargin Yu.F., Zhereb L.A. et al. Stable and metastable phase equilibria in the $\mathrm{Bi}_{2} \mathrm{O}_{3}-\mathrm{BiPO}_{4}$ system // Inorganic Materials. 2003. V. 39. № 8. P. 859-862].

7. Wignacourt J.P., Drache M., Conflant P. et al. New phases in $\mathrm{Bi}_{2} \mathrm{O}_{3}-\mathrm{BiPO}_{4}$ system. 1 . Description of phase diagram // J. Chim. Phys. 1991. V. 88. P. 1933 - 1938.

8. Wignacourt J.P., Drache M., Conflant P. et al. New phases in $\mathrm{Bi}_{2} \mathrm{O}_{3}-\mathrm{BiPO}_{4}$ system. 2. Structure and electrical properties of sillenite type solid solution // J. Chim. Phys. 1991. V. 88. P. $1939-1949$.

9. Mauvy F., Launay J.C., Darriet J. Synthesis, crystal structures and ionic conductivities of $\mathrm{Bi}_{14} \mathrm{P}_{4} \mathrm{O}_{31}$ and Bi50V4O85. Two members of the series $\mathrm{Bi}_{18-4 \mathrm{~m}} \mathrm{M}_{4 \mathrm{~m}} \mathrm{O}_{27+4 \mathrm{~m}}(\mathrm{M}=\mathrm{P}, \mathrm{V})$ related to the fluoritetype structure // J. Solid State Chem. 2005. V. 178. Р. 2015 - 2023.

10. Денисов В.М., Денисова Л.Т., Саблина К.А. и др. Высокотемпературная теплоемкость $\mathrm{Cu}_{5} \mathrm{Bi}_{2} \mathrm{~B}_{4} \mathrm{O}_{14} / /$ ДАН. 2013. Т. 450. № 4. С. 406 - 407. [Denisov V.M., Denisova L.T., Sablina K.A. and el. High-temperature specific heat of $\mathrm{Cu}_{5} \mathrm{Bi}_{2} \mathrm{~B}_{4} \mathrm{O}_{14} / /$ Doklady Physics. 2013. V. 58. № 6. P. 217 - 218].

11. Денисова Л.Т., Каргин Ю.Ф., Чумилина Л.Г. и др. Высокотемпературная теплоемкость $\mathrm{Sc}_{2} \mathrm{Cu}_{2} \mathrm{O}_{5} / /$ Неорган. материалы. 2014. Т. 50. № 5. С. 522 - 524. [Denisova L.T., Kargin Yu.F., Chumilina L.G. and el. High-temperature heat capacity of $\mathrm{Sc}_{2} \mathrm{Cu}_{2} \mathrm{O}_{5} / /$ Inorganic Materials. 2014. V. 50. №. 5. P. $482-484]$.

12. Maier, Chas G., and K. K. Kelley. An equation for the representation of high-temperature heat content data1 // Journal of the American chemical society 1932. V.54. №8 P. 3243-3246.

13. Скуратов С.М., Колесов В.П., Воробьев В.Ф. Термохимия. М.: Изд-во МГУ, 1966. Ч. ІІ. 434 c. [Skuratov, S.M., Kolesov, V.P., and Vorob'ev, V.F. Termokhimiya (Thermochemistry). Moscow: Mosk. Gos. Univ., 1966, part 2. 434 p.] 